MITSUBISHI ELECTRIC RESEARCH LABORATORIES

http://www.merl.com

\title{
Iterative Linear-Programming-Based Route Optimization for Cooperative Networks
}

\author{
Stark Draper, Lingjia Liu, Andreas Molisch, Jonathan Yedidia
}

TR2008-009 March 2008

\begin{abstract}
In this paper we develop linear-programming (LP) based route optimization techniques for networks of relays that employ mutual-information accumulation at the physical layer. Motivated by applications to unicast transmission in ultra wideband communications we concentrate on the regime where each node has a fixed bandwidth and transmission power. Our goal is to find the cooperative route that minimizes the source-to-destination transmission duration subject to a sum-energy constraint. We tackle this problem by solving a sequence of LP-based route optimizations under increasingly tight energy constraints, revealing a trade-off between energy consumption and delay. An initial route is found by "flooding" the network, resulting in the smallest delay, but largest energy consumption. Successive routes are found by initializing the LP with the optimum route found at the (slightly higher) previous energy constraint. Through this iterative procedure we explore a massive parameter space to find locally (and often globally) optimum solutions very efficiently. We illustrate our method for a network consisting of 50 nodes and compare the results to classic routing approaches. We comment on the applicability of our framework to other bandwidth and energy constraints, objective functions, and to multicasting.
\end{abstract}

Proc. International Zurich Seminar on Communications, March 2008

\footnotetext{
This work may not be copied or reproduced in whole or in part for any commercial purpose. Permission to copy in whole or in part without payment of fee is granted for nonprofit educational and research purposes provided that all such whole or partial copies include the following: a notice that such copying is by permission of Mitsubishi Electric Research Laboratories, Inc.; an acknowledgment of the authors and individual contributions to the work; and all applicable portions of the copyright notice. Copying, reproduction, or republishing for any other purpose shall require a license with payment of fee to Mitsubishi Electric Research Laboratories, Inc. All rights reserved.
} 



\title{
Iterative Linear-Programming-Based Route Optimization for Cooperative Networks
}

\author{
Stark C. Draper* ${ }^{*}$, Lingjia Liu ${ }^{\dagger}$, Andreas F. Molisch ${ }^{\ddagger \S}$, and Jonathan S. Yedidia ${ }^{\ddagger}$ \\ * Dept. of ECE, University of Wisconsin, Madison, WI 53706, E-mail: sdraper@ece.wisc.edu \\ $\dagger$ Dept. of ECE, Texas A\&M University, College Station, TX 77843, E-mail: lingliu@ece.tamu.edu \\ $\ddagger$ Mitsubishi Electric Research Laboratories, Cambridge, MA 02139, Email: \{draper, molisch, yedidia\}@merl.com \\ $\S$ Lund University, Sweden
}

\begin{abstract}
In this paper we develop linear-programming (LP) based route optimization techniques for networks of relays that employ mutual-information accumulation at the physical layer. Motivated by applications to unicast transmission in ultra wideband communications we concentrate on the regime where each node has a fixed bandwidth and transmission power. Our goal is to find the cooperative route that minimizes the sourceto-destination transmission duration subject to a sum-energy constraint. We tackle this problem by solving a sequence of LP-based route optimizations under increasingly tight energy constraints, revealing a trade-off between energy consumption and delay. An initial route is found by "flooding" the network, resulting in the smallest delay, but largest energy consumption. Successive routes are found by initializing the LP with the optimum route found at the (slightly higher) previous energy constraint. Through this iterative procedure we explore a massive parameter space to find locally (and often globally) optimum solutions very efficiently. We illustrate our method for a network consisting of 50 nodes and compare the results to classic routing approaches. We comment on the applicability of our framework to other bandwidth and energy constraints, objective functions, and to multicasting.
\end{abstract}

\section{INTRODUCTION}

A number of emerging applications involve routing delaysensitive data over wireless communication networks. In parallel, physical-layer methodologies have been developed that exploit cooperative relaying among nodes forming an adhoc network. While the optimum route strongly depends on the physical-layer technique used, often routing and physicallayer design are treated separately. We present a joint design where, at the physical layer, relaying nodes employ mutualinformation accumulation.

At the physical layer, most routing papers that consider relaying use either maximum-ratio transmission (virtual beamforming), e.g., [1], [2], [9], [11] or maximum-ratio combining, e.g., [12]. ${ }^{1}$ Maximum-ratio combining/transmission can be characterized as energy-accumulation. As the number of transmitters increase throughput scales as $\log \left[1+\sum_{i} P_{i} / N_{0}\right]$ where $P_{i} / N_{0}$ is the receive signal-to-noise ratio (SNR). In contrast, in this paper we consider networks that employ mutualinformation accumulation [3], [13]. In mutual-information accumulation transmitters send different symbols across parallel channels and throughput scales as $\sum_{i} \log \left[1+P_{i} / N_{0}\right]$. While at very low SNRs energy and information accumulation result in the same throughput, at high SNRs the gains of mutualinformation accumulation are significant. A related problem of route optimization while accumulating "soft" information

\footnotetext{
${ }^{1}$ For relaying in non-routing contexts see, e.g., [8], [10], [15], [16].
}

is explored in [17]. While the framework considered there is philosophically similar to ours, the details differ (power optimization there, degrees-of-freedom allocation here) and [17] does not consider the decoding-order optimization that sits at the center of our investigation. Instead, they identify good baselines routes and optimize power allocations for them.

In this paper we design minimum-delay routing algorithms for networks where transmitters operate under fixed-power and fixed-bandwidth constraints. Our choice of constraints is motivated by applications in ultra wideband communication where available bandwidth and transmit power are determined by frequency regulators [4]. Furthermore, constraints on the spreading factor are imposed by limits on hardware complexity as well as requirements of communications standards [14]. Our solution entails solving a sequence of linear programs (LPs). The fundamental setup is closely connected to the one we investigate in [7]. However, it differs in the key respect that [7] considers a network-wide sum-bandwidth constraint, while the current paper assumes a fixed bandwidth constraint on each node. As a consequence, the approach and solution differ in a number of important aspects. First, [7] shows that, under a sum-bandwidth constraint and when transmission power is constant across nodes, the minimumdelay and minimum-energy routes are the same. In contrast, under per-node bandwidth constraints there is a trade-off between energy consumption and transmission delay. Second, the existence of an energy/delay trade-off makes the routing algorithm more involved. In particular, we identify a good algorithmic initialization to be the "flooding" route. This is the easily-found minimum-delay route when no energy constraints are imposed. We then solve a sequence of LPs, iteratively tightening the energy constraint until the target energy is met.

We present the system model in Section II. In Section III we develop our algorithms. We detail simulation results in Section IV and conclude in Section V.

\section{SYSTEM MODEL}

We focus on unicast routing in a network consisting of $N+1$ nodes. The objective is to convey a data packet of $B$ bits from source to destination in the minimum time under energy and bandwidth constraints. The $N-1$ non-source/destination nodes are relays. They may take an active role in transmission or may remain silent for the duration. Nodes are half-duplex: they can either receive or transmit but cannot do both simultaneously.

The $i$ th node transmits at a fixed power spectral density (PSD) of $P_{i}$ (joules $\left./ \mathrm{sec} / \mathrm{Hz}\right)$, uniform across its transmission 
band. The propagation channel between each pair of nodes is modeled as frequency-flat and block-fading. The channel power gain between the $i$ th and the $j$ th nodes is denoted $h_{i, j}$. The spectral efficiency from node $i$ to node $j$ is

$$
C_{i, j}=\log _{2}\left[1+\frac{h_{i, j} P_{i} W_{i}}{N_{0} W_{i}}\right]=\log _{2}\left[1+\frac{h_{i, j} P_{i}}{N_{0}}\right] \text { bits/s/Hz, }
$$

where $N_{0} / 2$ denotes the PSD of the white Gaussian noise, and $W_{i}$ is the transmission bandwidth of node $i$. Energy and bandwidth constraints are discussed in detail in Sec. III.

Transmitters use independently-generated ideal codes and transmit over non-interfering channels. The independence assumption is crucial to mutual-information accumulation. If the same code were used by each transmitter, the system would be implementing energy-accumulation rather than mutual information accumulation. Nodes listen to all transmissions and combine all receptions to recover the data message. The only requirement for decoding is that the total received mutual information (summed over all transmitting nodes) exceeds $B$ bits [13]. This is what we mean by ideal codes.

\section{Routing \& Resource-AllocAtion}

To optimize the route we iterate between solving two subproblems. On the one hand we identify a "decoding order" that comprises a subset of the $N+1$ nodes. These nodes are labelled in the order in which they decode the data packet. This is the same order in which they can come on-line as relays. On the other hand, given a particular decoding order we determine the optimal resource allocations (time, energy, bandwidth). The resource allocation problem can be expressed as a LP. The solution of this LP is then used to refine the decoding order. By iterating between these two sub-problems we quickly find a very good locally optimum (and often globally optimum as we have verified on small networks) route and resource allocation, even for very large networks.

\section{A. Optimizing resource allocation for fixed decoding order}

Of the $N+1$ nodes consider a subset of $L+1$ that form a particular decoding order. Without loss of generality label nodes according to their (current) decoding order: $0,1,2, \ldots, L$ where node 0 is the source and node $L$ is the destination.

Denote the time at which node $i$ decodes the message as $T_{i}$ with $T_{0}=0$. The source-destination transmission duration is $T_{L}$. Instead of working with the $T_{i}$ we work with the internode decoding delays $\Delta_{i}=T_{i}-T_{i-1}$ for $1 \leq i \leq L$. Message transmission consists of $L$ phases. The $i$ th phase is of duration $\Delta_{i}$ and is characterized by the fact that at the end of the phase the first $i$ nodes have decoded the message. We refer to each phase as a "time-slot". Time-slots are not of pre-set or equal lengths, but rather are solved for as we discuss next.

For a given decoding order, our objective is to minimize

$$
T_{L}=\sum_{i=1}^{L} \Delta_{i}
$$

subject to: (i) $\Delta_{i} \geq 0$ for all $i$, (ii) node $i$ must decode by time $T_{i}=\sum_{l=1}^{i} \Delta_{l}$, (iii) the energy constraint, and (iv) degree-offreedom constraints.
The $L$ decoding constraints are

$$
\sum_{i=0}^{k-1} \sum_{j=i+1}^{k} A_{i, j} C_{i, k} \geq B \quad \text { for all } k \in\{1,2, \ldots, L\}
$$

where

$$
A_{i, j} \geq 0 \text { for all } i \in\{0,1, \ldots, L-1\}, j \in\{1,2, \ldots, L\} .
$$

The $A_{i, j}$ are the degrees-of-freedom, i.e., the time-bandwidth product (or "area" in $\mathrm{sec} \cdot \mathrm{Hz}$ ) used by the $i$ th node in the $j$ th time slot. Recall that $C_{i, k}$ is the spectral efficiency in $\mathrm{bits} / \mathrm{sec} / \mathrm{Hz}$. The information accumulated by the $k$ th node must exceed $B$ bits by the end of the $k$ th slot for the node to decode. Only the first $k-1$ nodes contribute to this sum. The remaining nodes have not yet decoded so cannot yet transmit.

Not all $N+1$ nodes need be included in the decoding order, only a subset of cardinality $L+1$. If, for instance, one node (neither source nor destination) is far from the rest (or masked by a building), then including its decoding constraint in the set (2) would only increase $T_{L}$. As we discuss when we present the "swapping" algorithm that improves the decoding order, nodes can be dropped from the order yielding an $L<N$.

In this paper the sum-energy allocation is constrained as

$$
\sum_{i=0}^{L-1} \sum_{j=1}^{L} A_{i, j} P_{i}=\sum_{i=0}^{L-1} \sum_{j=i+1}^{L} A_{i, j} P_{i} \leq E_{\mathrm{T}},
$$

where the equality holds because $A_{i, j}=0$ for $j \leq i$. This is true since node $i$ has not decoded until the end of slot $i$ and thus can only transmit (and therefore would only be allocated positive bandwidth) in slots $i+1, \ldots, L$.

We constrain nodes to transmit over parallel channels of equal bandwidth $W_{\text {node }}$. Each node is allocated at most a single channel in each time slot, thus

$$
A_{i, j} \leq \Delta_{j} W_{\text {node }} \text { for all } \quad \begin{aligned}
& i \in\{0,1, \ldots L-1\} \\
& j \in\{1,2, \ldots, L\}
\end{aligned} .
$$

As is discussed further in [6] the LP framework can accommodate a variety of other resource constraints and objective functions. We provide some examples now. The sum-energy constraint (3) can be replaced with the per-node constraints $\sum_{j=i+1}^{L} A_{i, j} P_{i} \leq E_{i}$ for all $i \in\{1,2, \ldots, L\}$. The pernode bandwidth constraints (4) can be replaced with the sum-bandwidth allocation $\sum_{i=0}^{j-1} A_{i, j} \leq \Delta_{j} W_{\mathrm{T}}$ for all $j \in$ $\{1,2, \ldots, L\}$ where $W_{\mathrm{T}}$ is the system-wide bandwidth allocation. The objective function (1) can be refocused on the sumenergy minimization $\sum_{i=0}^{L-1} \sum_{j=i+1}^{L} A_{i, j} P_{i}$ subject to end-toend delay constraints $\sum_{i=1}^{L} \Delta_{i} \leq \tau_{\text {tot }}$. Alternately, one might want to minimize the time-bandwidth footprint. This would allow for parallel transmissions (between different sourcedestination pairs), or could be used to minimize inter-network interference (if multiple networks are operating in the same area). In this case one would choose the objective function to be $\sum_{i=0}^{L-1} \sum_{j=1}^{L} A_{i, j}$ subject to delay and energy constraints.

\section{$B$. Optimizing the decoding order}

The linear program stated above solves the resourceallocation problem. However, it leaves open the question of how to determine the best decoding order. In a network of 
$N+1$ nodes there are $1+\sum_{l=1}^{N-1} \frac{(N-1) !}{(N-1-l) !}$ distinct orderings. For a 50 node network this is approximately $1.6 \times 10^{63}$. Exhaustive search quickly exceeds computational capabilities.

We introduce an algorithm that iteratively improves the decoding order by exploiting the characteristics of the LP solution obtained in Section III-A. The algorithm progressively reduces the delay by refining the decoding order until a locally optimum ordering is obtained. While in general we obtain a local minimum, for small networks (of, e.g., 9 nodes, where we can exhaustively search all orderings) we almost always reach the global optimum. Additionally, we can try a number of different initializations to avoid particularly bad local minima.

Consider an arbitrary decoding order. Define

$$
\mathrm{x}^{*}=\left[\Delta_{1}^{*}, \ldots, \Delta_{L}^{*}, A_{0,1}^{*}, A_{0,2}^{*}, \ldots A_{0, L}^{*}, A_{1,2}^{*}, \ldots, A_{L-1, L}^{*}\right]
$$

to be the corresponding LP solution. Denote the corresponding decoding delay as $T_{L}^{*}=\sum_{i=1}^{L} \Delta_{i}^{*}$. The following theorem proved in [6] holds when subject to either sum- or per-node bandwidth and either sum- or per-node energy constraints.

Theorem 1. If $\Delta_{i}^{*}=0$, use $T_{L}^{* *}$ to denote the optimum decoding delay (under the same energy and bandwidth constraints) of the "swapped" decoding order:

$$
\begin{array}{ll}
{[0, \ldots, i-2, i, i-1, i+1, \ldots, L]} & \text { if } i \leq L-1 \\
{[0, \ldots, L-2, L]} & \text { if } i=L
\end{array}
$$

Then $T_{L}^{* *} \leq T_{L}^{*}$.

We now state the iterative route optimization algorithm.

\section{Algorithm 1:}

1) Start with an initial decoding order.

2) Use the linear program of Section III-A to solve for the parameters of the minimum-delay solution.

3) Based on Theorem 1 adapt the decoding order to find an ordering whose minimum-delay solution is upper bounded by the delay of the current solution:

a) If $\Delta_{i}=0$ for a single $i$, swap nodes $i$ and $i-1$.

b) If $\Delta_{i}=\Delta_{j}=0$ for $i<j-1$ swap both nodes $i$ and $i-1$ and $j$ and $j-1$. If $\Delta_{i}=\Delta_{i+1}=0$ swap only $i$ and $i-1$.

c) If the node $L-1$ is swapped with node $L$, drop (the former) node $L-1$ from the order entirely. The resulting order constraints only $L-1$ nodes.

4) Repeat steps 2)-3) until an ordering is obtained with an associated set of parameters $\mathrm{x}^{*}$ satisfying $\Delta_{i}^{*}>0$ for all $i$. At this point terminate the algorithm.

If we initialize Algorithm 1 at the target sum-energy constraint and an arbitrary decoding order we typically find that $\Delta_{i}^{*}=0$ for multiple $i$. It is then not clear what change to the ordering should be made. We propose the following,

\section{Algorithm 2:}

1) Initialize Algorithm 1 with the "flooding" order/energy.

2) Run Algorithm 1.

3) If the energy constraint equals the target, terminate the algorithm. Otherwise, tighten the energy constraint and use the new route for initialization in step 2).

Algorithm 2 solves a sequence of route optimizations using Algorithm 1 under tighter and tighter energy constraints until

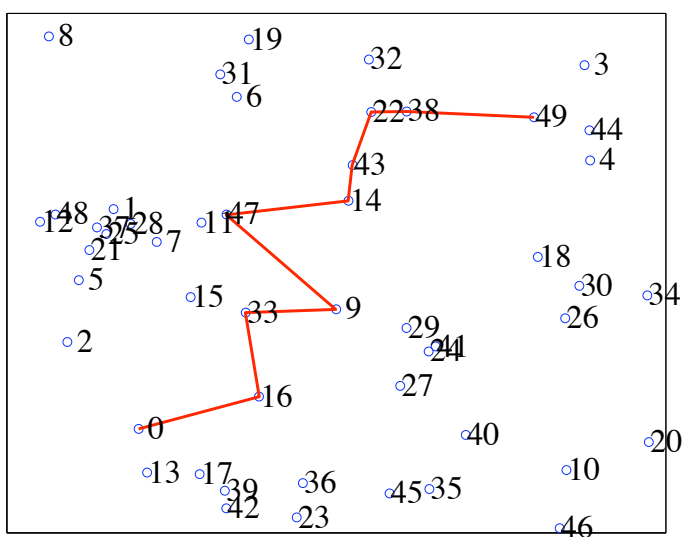

Fig. 1. Fifty node network with minimum-energy cooperative routing shown.

the target energy budget is met. The optimized route found under one energy constraint is used to initialize Algorithm 1 under the next, slightly tighter, energy constraint. As with most non-linear iterative optimization routines, the choice of step size is important. In Algorithm 2 the step size corresponds to the increment by which the energy constraints are tightened. Ideally, the energy constraints are tightened only enough that a single $\Delta_{i}^{*}=0$. This can typically be accomplished by making the increment small or dynamically choosing the increment. If the energy constraint is over-tightened (multiple $\Delta_{i}^{*}=0$ ), one can reduce the increment slightly and re-solve the LP.

The "flooding" order is the optimum order when the energy constraint is fully relaxed. This allows nodes unlimited energy consumption and achieves the minimum possible transmission delay. This delay and the corresponding order is easily calculated as follows. The source node starts transmitting at time 0 . Other nodes join in and begin transmitting as soon as they decode. All nodes continue to transmit until the destination decodes. The order in which nodes come on-line as transmitters is the "flooding" order.

We note that we can apply our algorithms to cooperative routing for multicasting. One simply never drops any of the (now multiple) "destination nodes" from the decoding order.

\section{ILLUSTRATIVE RESUlTS}

In this section we provide illustrative results for the network presented in Fig. 1. The source node 0 is located at $[0.2,0.2]$ and the destination node 49 is located at $[0.8,0.8]$. The remaining nodes are placed randomly according to a uniform distribution in the unit square. The labelling of nodes in Fig. 1 is for reference purposes only - it does not correspond to a particular decoding order. To give the reader a strong sense of the relationship between geometry and channel strength we present the case where the channel gain $h_{i, j}$ between nodes $i$ and $j$ is deterministically related to the distance $d_{i, j}$ between them as $h_{i, j}=\left(d_{i, j}\right)^{-2}$. In the following results $B=28.9$ (20 nats), $N_{0} / 2=1, W_{\text {node }}=1$, and $P_{i}=1$ for all $i$.

All nodes except 3,4 , and 44 participate in the flooding routing: $\quad[0,13,17,39,42,16,2,36,23,15, \ldots, 20,32,34,8,49]$. As the energy constraint is tightened, delay increases until, at some minimum energy, the network becomes disconnected. The network's energy-delay trade off is plotted in Fig. 2. 


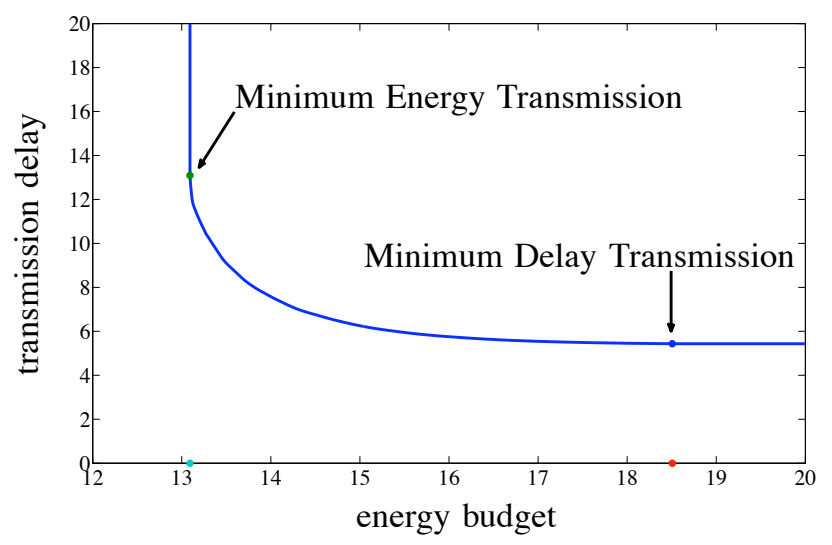

Fig. 2. Energy/delay trade off for the fifty node network.

Typically, as the energy budget is decreased, nodes with weaker connectivity to the destination go off-line and only nodes with stronger connectivity continue to transmit. In Fig. 1 the minimum-energy route $[0,16,33,9,47,14,43,22,38,49]$ is depicted by the connected nodes. Note that in the figure we only indicate nodes that transmit. There are nodes in the minimum-energy decoding order that do not transmit.

To quantify the performance gains of our approach we establish a baseline non-cooperative strategy for comparison. This is a multi-hop approach where only one node transmits at a time. The route is selected using Dijkstra's shortest path algorithm [5] under the assumption that each node listens only to its immediate predecessor. For the network of Fig. 1 this route is $[0,9,49]$. We also consider a hybrid strategy that uses the route obtained by Dijkstra's algorithm as above, but where nodes to perform mutual information accumulation. That is, nodes listen to all previous transmissions. (This latter strategy is akin to one of the proposals in [17].) By studying both cases we establish the fractional performance improvement due to the use of mutual-information accumulation, and the further gain due to the close coupling of route optimization with the physical-layer technique being used.

It is important to note that under per-node bandwidth constraints the peak bandwidth usage of the non-cooperative scheme is limited to the transmission band of a single node $W_{\text {node }}$. In contrast, in the cooperative setting many nodes may transmit simultaneously. In general, while the peak bandwidth usage of the cooperative strategy exceeds that of the noncooperative strategy, the total time-bandwidth usage is lower.

One operating point where the cooperative and noncooperative strategies are fully comparable is when the former is at the minimium energy. We show in [6] that at the minimum energy the optimum cooperative route has only one node transmitting in any given time slot. The peak bandwidth usage of cooperative and non-cooperative strategies are therefore identical. For the network of Fig. 1 the cooperative routing algorithm proposed in this paper results in a minimumenergy transmission duration of 13.09 seconds. In contrast, when the Dijkstra route is used without mutual-information accumulation the transmission duration is 21.47 seconds, while when the Dijkstra route is used with mutual-information accumulation the duration is 16.51 seconds. Respectively, these transmission durations are $64 \%$ and $26 \%$ longer than that resulting from our routing. Further note that since in this example the transmit PSD is constant across nodes, and only one node is transmitting at any given time, energy usage is proportional to transmission delay. Therefore substantial delay and energy savings result both from the use mutualinformation accumulation and from route optimization.

\section{SUMMARY AND CONCLUSIONS}

In this paper we pose the problem of routing in cooperative relay networks that use mutual-information accumulation. We split the problem into one of finding the best decoding order and one of finding the best resource allocation given a decoding order. As our solution is based on solving a sequence of linear programs, it is quite efficient. The approach presented in this paper is a step towards practically realizing cooperative communications in large networks. Future work will focus on algorithms that are suitable for imperfect channel state information and the impact of non-ideal codes and hardware.

Acknowledgements: We thank Dr. Neelesh Mehta for useful discussions, and Dr. Jin Zhang, Dr. Kent Wittenburg and Dr. Joseph Katz for their support and encouragement.

\section{REFERENCES}

[1] M. Abdallah and H. Papadopoulos. Beamforming algorithms for decodeand-forward relaying in wireless networks. In Proc. Conf. Inform. Sciences \& Systems, 2005.

[2] G. Barriac, R. Mudumbai, and U. Madhow. Distributed beamforming for information transfer in sensor networks. In Int. Symp. Inform. Proc. Sensor Networks, page 81, 2004.

[3] J. Castura and Y. Mao. Rateless coding over fading channels. IEEE Commun. Lett., 10:46-48, 2006.

[4] Federal Communications Commission. First report and order 02-48, April 2002.

[5] T. H. Cormen, C. E Leiserson, and R. L Rivest. Introduction to Algorithms. MIT Press and McGraw-Hill, 2001.

[6] S. C. Draper, L. Liu, A. Molisch, and J. S. Yedidia. Routing in mutualinformation accumulating relaying networks. IEEE Trans. Inform. Theory, To be submitted.

[7] S. C. Draper, L. Liu, A. F. Molisch, and J. S. Yedidia. Routing in cooperative wireless networks with mutual-information accumulation. In Proc. Int. Conf. Commun., Beijing, August 2008.

[8] T. E. Hunter, S. Sanayei, and A. Nosratinia. Outage analysis of coded cooperation. IEEE Trans. Inform. Theory, 52:375-391, 2006.

[9] A. E. Khandani, J. Abounadi, E. Modiano, and L. Zheng. Cooperative routing in static wireless networks. IEEE Trans. Commun., 55:21852192, November 2007.

[10] J. N. Laneman, D. N. C. Tse, and G. W. Wornell. Cooperative diversity in wireless networks: Efficient protocols and outage behavior. IEEE Trans. Inform. Theory, 50:3062, 2004.

[11] R. Madan, N. B. Mehta, A. F. Molisch, and J. Zhang. Energy-efficient decentralized routing with localized cooperation suitable for fast fading. In Allerton Conf. on Communication, Control and Computing, 2007.

[12] I. Maric and R. D. Yates. Cooperative multihop broadcast for wireless networks. IEEE J. Select. Areas Commun., 22:1080-1088, 2004.

[13] A. F. Molisch, N. B. Mehta, J. S. Yedidia, and J. Zhang. Performance of fountain codes in collaborative relay networks. IEEE Trans. Wireless Commun., 6:4108-4119, 2007.

[14] A. F. Molisch, P. Orlik, Z. Sahinoglu, and J. Zhang. UWB-based sensor networks and the IEEE 802.15.4a standard - a tutorial. In Chinacomm, 2006.

[15] A. Sendonaris, E. Erkip, and B. Aazhang. User cooperation diversitypart I: System description. IEEE Trans. Commun., 51:1927, 2003.

[16] A. Stefanov and E. Erkip. Cooperative coding for wireless networks. IEEE Trans. Commun., 52, 2004.

[17] Z. Yang and A. Host-Madsen. Routing and power allocation in asynchronous Gaussian multiple-relay channels. EURASIP J. Wireless Commun. Networking, pages 1-11, 2006. 